Aleksandar S. Nikolić

Univerzitet u Novom Sadu

Filozofski fakultet

Student doktorskih studija

aleksandar.nikolic@ff.uns.ac.rs doi: 10.19090/zjik.2020.59-71

UDK 811.112.2:811.163.41]'373.7

stručni naučni rad

\title{
KONTRASTIVE ANALYSE DEUTSCHER PHRASEOLOGISMEN IM SEMANTISCHEN FELD „REDEN“ UND „SCHWEIGEN“ UND IHRER ÄQUIVALENTE IM SERBISCHEN
}

Der folgende Beitrag behandelt deutsche Phraseologismen aus dem semantischen Feld „reden“ und „schweigen“ und ihre entsprechenden Äquivalente im Serbischen. Ziel der Arbeit ist es, mithilfe der Methode der interlingualen kontrastiven Analyse auf alle morphologischen, morphosyntaktischen und lexikalischen Gemeinsamkeiten und Unterschiede zwischen Phraseologismen beider Sprachen aufmerksam zu machen, welche auf das genannte semantische Feld zurückzuführen sind, wobei Deutsch als Ausgangssprache bzw. L1 und Serbisch als Zielsprache bzw. L2 zu betrachten ist. Dabei kommen nicht nur Beispiele mit den Komponenten „reden“ und „schweigen“ infrage, sondern alle Phraseologismen, aus deren Bedeutung heraus „reden“ und „schweigen“ erschlossen werden können. Das Korpus bilden insgesamt 49 Beispiele, welche verschiedenen einsprachigen und zweisprachigen phraseologischen Wörterbüchern in deutscher, serbischer, serbokroatischer und kroatischer Sprache entnommen wurden. Die Ergebnisse dieser Analyse sollten im Germanistikstudium, DaF-Unterricht und in der Tätigkeit von Übersetzern ihre Verwendung finden.

Schlüsselwörter: reden, schweigen, Phraseologie, Deutsch, Serbisch

\section{EINLEITUNG}

Im Phraseologismus Reden ist Silber, Schweigen ist Gold sind die zwei Grundbegriffe sprachlicher Äußerungen bzw. Nichtäußerungen, welche in diesem Beitrag behandelt werden, enthalten. Entweder man redet oder man schweigt und das wird oftmals durch unterschiedliche, variantenreiche Phraseologismen ${ }^{1}$

\footnotetext{
${ }^{1}$ Burger definiert Phraseologismen als Ausdrücke, die aus mehr als einem Wort bestehen und die dem Rezipienten in genau dieser Kombination - eventuell mit alternativen Varianten - bekannt sind, wobei die einzelnen Bestandteile als Komponenten zu betrachten sind (Burger, 2003: 11). Aus der Definition erschließen sich bereits zwei Merkmale, und zwar die Polylexikalität und die Festigkeit, welche Burger den sog. Phraseologismen im weiteren Sinne zuschreibt, während Phraseologismen im engeren
} 
ausgedrückt. Das Ziel dieser Arbeit ist es, Phraseologismen im Deutschen und Serbischen zu kontrastieren und sie in Bezug auf morphologische, morphosyntaktische und lexikalische Gemeinsamkeiten und Unterschiede zu untersuchen, um schließlich deren Äquivalenzgrad feststellen zu können.

1.1 Das Korpus besteht aus Phraseologismen, die folgenden Wörterbüchern entnommen wurden: Wer hat den Teufel an die Wand gemalt? vom Dudenverlag, Deutsch-serbokroatisches phraseologisches Wörterbuch von Pavica Mrazović und Ružica Primorac, Hrvatsko-njemački frazeološki rječnik und Frazeološki rječnik hrvatskoga ili srpskog jezika von Josip Matešić, Srpskonemački prevodni frazeološki rečnik von Božinka Petronijević, Lexikon der sprichwörtlichen Redensarten von Lutz Röhrich und Frazeološki rečnik srpskog jezika von Đorđe Otašević. Bei der Korpuserstellung wurden nicht ausschließlich Phraseologismen entnommen, welche die Komponenten „reden“ und schweigen“ beinhalten, sondern alle, aus derer Semantik heraus „reden“ oder „schweigen“ erschlossen werden konnten. Die dabei entnommenen 49 Beispiele erheben keinen Anspruch auf Vollständigkeit, sondern stellen lediglich eine Grundlage für zukünftige Untersuchungen in diesem Bereich dar.

\section{KONTRASTIVE PHRASEOLOGIE UND ÄQUIVALENZ}

Korhonen unterscheidet im Kontext der kontrastiven Phraseologie - einer Teildisziplin der kontrastiven Linguistik - zwischen einer intra- und interlingualen bzw. zwischen einer kontrastiven Phraseologie im weiteren und einer im engeren Sinne, wobei die erste Phraseologismen in einer Sprache untersucht und die zweite Phraseologismen verschiedener Sprachen einander entgegensetzt (Korhonen,

Sinne noch das Merkmal der Idiomatizität (eine Eigenschaft sprachlicher Ausdrücke, die darin besteht, dass man ihre Bedeutung nicht aus der Bedeutung ihrer einzelnen Bestandteile herleiten kann) hinzugefügt wird. Diese Konstruktionen bezeichnet er als Idiome. Christine Palm erwähnt die Idiomatizität im Kontext einer Reinterpretation oder semantischen Transformation bestimmter Komponenten in einem Phraseologismus (Palm, 1997: 9). Fleischer äußert ähnliche Ansichten in Bezug auf die Merkmale, allerdings fügt er zusätzlich die Eigenschaft der Reprodruzierbarkeit hinzu - d. h., dass Phraseologismen durch mehrere Wiederholungen in unserem Gedächtnis gespeichert werden (Fleischer, 1997: 29-30). In dieser Arbeit werden die von Burger für Phraseologismen im weiteren Sinne genannten Merkmale der Polylexikalität und Festigkeit als Grundlage zur Korpuserstellung genommen - d. h., dass das Fehlen der Idiomatizität nicht zu einer Ausschließung von Beispielen führt. Der von Burger genannte Begriff Idiom wird in dieser Arbeit mit dem Terminus Phraseologismus gleichgesetzt. 
2007: 574-577). In dieser Entgegensetzung zweier Sprachsysteme ist der Terminus der Äquivalenz zu nennen, wobei es sich in erster Linie um die Hervorhebung von Gemeinsamkeiten und Unterschieden handelt, die im Laufe eines Übersetzungsprozesses entstehen. In diesem Zusammenhang wird meistens zwischen einer Voll-, Teil- und Nulläquivalenz unterschieden, wobei Burger noch eine semantische Äquivalenz nennt (Burger et al., 1982: 289). Für die Bedürfnisse dieses Beitrags erwies sich die Klassifizierung von Pavica Mrazović am günstigsten.

2.1 Pavica Mrazović zerlegt in ihrer Klassifizierung die Teiläquivalenz in eine mit lexikalischen, morphosyntaktischen und lexikalischen und morphosyntaktischen Unterschieden, und nennt neben der Voll- und Nulläquivalenz schließlich noch „falsche Freunde“, sodass ihre Unterteilung letztendlich sechs Untergruppen des phraseologischen Äquivalenzverhältnisses zählt (Mrazović: 1964-65: 293):

1) Die Volläquivalenz - Es kommt zu einer vollständigen lexikalischen, morphosyntaktischen und semantischen Übereinstimmung, wie z. B.:

jemandem den Mund stopfen - začepiti nekome usta

2) Falsche Freunde - Phraseologismen, die strukturell, formell und lexikalisch übereinstimmen, jedoch semantisch nicht dieselbe Bedeutung tragen, wie z. B.:

sich an die Brust schlagen - busati se u grudi

Der deutsche Phraseologismus hat im Serbischen ein strukturelles phraseologisches Äquivalent in busati se u grudi. Er bezieht sich hierbei auf Situationen, in denen man etwas bereut, oder sich Vorwürfe macht. Der serbische Phraseologismus bedeutet hingegen, dass sich jemand in den Mittelpunkt stellt, bzw. heldenhaft erscheinen möchte.

3) Teiläquivalenz mit morphosyntaktischen Unterschieden - Die Phraseologismen gleichen einander auf lexischer und semantischer Ebene, während die morphosyntaktische Ebene Unterschiede aufweist, wie z. B.:

das ist zum Schießen - to je da pukně̌ od smeha

4) Teiläquivalenz mit lexikalischen Unterschieden: schweigen wie ein Grab - ćutati kao zaliven

5) Teiläquivalenz mit lexikalischen und morphosyntaktischen Unterschieden:

um den heißen Brei herumreden - obilaziti kao kiša oko Kragujevca 
6) Nulläquivalenz: Phraseologismen der Ausgangssprache entspricht kein Phraseologismus der Zielsprache:

reden wie ein Blinder von der Farbe - govoriti besmislice

\section{KORPUSANALYSE ${ }^{2}$}

Das analysierte Korpus besteht aus insgesamt 49 Phraseologismen aus dem semantischen Feld „reden“ und „schweigen“, die verschiedenen einsprachigen und zweisprachigen Wörterbüchern entnommen wurden. Die Beispiele wurden nach der Klassifizierung von Pavica Mrazović in Gruppen eingeteilt, wobei $\mathrm{zu}$ erwähnen ist, dass kein einziges Beispiel der Äquivalenzgruppe „falsche Freunde“ zugeordnet werden konnte. Es wurden auch keine Beispielpaare gefunden, in denen der Unterschied ausschließlich morphosyntaktischer Natur ist. Bei morphosyntaktischen Abweichungen war immer auch eine lexikalische Differenz präsent. In der nachfolgenden Analyse wird auch die Semantik der einzelnen Phraseologismen erläutert und bei Entgegensetzungen, die eine Null- oder Teiläquivalenz nach sich ziehen, werden auch buchstäbliche Übersetzungen aus dem Deutschen ins Serbische geboten. Aus Lesbarkeitsgründen wurden folgende Abkürzungen verwendet:

Bd.: Bedeutung und nichtphraseologische Periphrase

W: die wörtliche Übersetzung des deutschen Phraseologismus

Bsp.: Beispielsatz

3.1 Die Analyse ergab insgesamt 15 Beispiele von vollkommener Äquivalenz, d. h., dass in diesen Fällen deutsche und serbische Phraseologismen in den Bereichen Lexik, Morphosyntax und Semantik übereinstimmten, wovon elf dem semantischen Feld „reden“ und drei dem semantischen Feld „schweigen“ zuzuschreiben sind. In einem Beispiel kamen beide Komponenten vor:

reden wie aufgezogen ${ }^{3}$ - govoriti kao navijen/bez prestanka

Bd.: Der Phraseologismus bezieht sich auf die Verhaltensweise von Menschen, die ununterbrochen reden.

\footnotetext{
${ }^{2}$ Wegen der begrenzten Länge des Beitrags konnten nicht alle Beispiele in den Beitrag aufgenommen werden.

${ }^{3}$ Der Phraseologismus Reden wie ein Wasserfall kommt in derselben Bedeutung vor.
} 
Bsp.: Ich konnte nichts sagen, er redet wie aufgezogen. - Nisam mogao ništa da kažem, priča kao navijen.

jemandem den Mund/das Maul stopfen ${ }^{4}$ - začepiti (zatvoriti) nekome usta

Bd.: Jemandes Rede absichtlich unterbrechen.

Bsp.: Hör auf zu reden oder ich werde dir den Mund stopfen. - Prestani da govoriš ili ću ti začepiti usta.

In einigen Fällen bot die Ausgangssprache mehrere Möglichkeiten, von denen nur eine im Vergleich mit dem Serbischen als vollkommenes Äquivalent angesehen werden konnte. Bei der Verwendung alternativer Möglichkeiten käme eine partielle Äquivalenz mit lexikalischen Unterschieden zustande. Solche Beispiele wurden jedoch als Beispiele für Volläquivalenz gedeutet.

- leeres Stroh dreschen/hohle Phrasen dreschen - mlatiti (lupati) praznu slamu

Bd.: Der Phraseologismus bezieht sich auf sinnlose Taten und sinnloses Gerede.

Bsp.: Er hat nichts Besonderes gesagt, nur leeres Stroh gedroschen. Nije rekao ništa posebno, samo je mlatio praznu slamu.

- eine spitze/scharfe/böse/giftige Zunge haben ${ }^{5}$ - biti oštar na jeziku/imati oštar jezik

Bd.: Eine meistens abwertende Charakterisierung von Menschen, die angriffslustig und rücksichtslos reden, ohne jegliche Hemmungen und ohne Rücksicht auf Konsequenzen oder die Gefühle anderer.

Bsp.: Was die mir alles ins Gesicht gesagt hat. Also diese Person hat eine äußerst scharfe Zunge. - Šta mi je sve rekla u lice. Baš ima oštar jezik.

- jemandem in die Rede/ins Wort fallen ${ }^{6}$ - upasti nekome u reč

Bd.: Jemanden unterbrechen, während er/sie redet.

\footnotetext{
${ }^{4}$ Umgangssprachlich werden die Nomen „Mund“/,Maul“ mitunter durch „Fresse“ ersetzt.

${ }^{5}$ Die Adjektive „,spitz“, „,böse“ und ,giftig“ finden im Serbischen kein Gegenstück, sodass in Fällen, in denen der Phraseologismus eine spitze/böse/giftige Zunge haben lautet, nur Teiläquivalenz besteht, und zwar mit lexikalischen Unterschieden (serb. imati šiljat/zao/otrovan jezik).

${ }^{6}$ Wenn in der deutschen Wortfolge das Lexem „Wort" vorkommt, besteht Volläquivalenz mit dem serbischen Phraseologismus. Bei der Variante jemandem in die Rede fallen (serb. upasti nekome u govor), handelt es sich um Teiläquivalenz mit lexialischen Unterschieden.
} 
Bsp.: Ich konnte keinen Satz zu Ende bringen, er ist mir immer ins Wort gefallen. - Nisam mogao nijednu rečenicu da završim do kraja, stalno mi je upadao u reč.

- $\quad$ Reden ist Silber, Schweigen ist Gold ${ }^{7}$ - govor je srebro, ćutanje je zlato

Bd.: Das bedeutet, das es in vielen Situationen besser ist, still zu sein, als zu reden.

Bsp.: Reden ist Silber, Schweigen ist Gold, sagt der Volksmund. - (Govor je srebro), ćutanje je zlato - kaže narod.

3.2 Für 16 der insgesamt 49 Beispiele konnten keinerlei Entsprechungen nachgewiesen werden, sodass in diesen Fällen Nulläquivalenz festgestellt wurde. Auch hier lies sich die dominante Präsenz des semantischen Feldes „reden“ erkennen, welchem elf Beispiele zugeordnet werden können.

- reden wie ein Blinder von der Farbe - govoriti besmislice

Bd.: Der Phraseologismus bezieht sich auf unqualifizierte Bemerkungen und Aussagen, die jemand macht, obwohl das Thema größtenteils unbekannt ist.

W: govoriti kao slepac o boji

Bsp.: Um Jugendlichen klarzumachen, dass Drogen schädlich sind, sollte man nicht nur wie der Blinde von der Farbe reden.

- der langen Rede kurzer Sinn - ukratko/sažeto/da skratim/evo šta je najvažnije/da ne dužim

Bd.: Der Phraseologismus hat bei Gesprächen die Funktion einer Floskel und signalisiert das Ende der Rede.

W: dugog govora kratki smisao

Bsp.: Ich musste in die Bank, dann in die Bibliothek und dann musste ich noch einkaufen. Langer Rede kurzer Sinn, ich hab die Arbeit nicht gemacht.

- große (endlose) Reden schwingen - mnogo pričati

Bd.: Dieser Phraseologismus beschreibt Menschen, die gerne prahlen.

W: zavitlati velike (beskonačne) govore/priče

Bsp.: Eigentlich kann er gar nichts. Er kann nur große Reden schwingen.

- $\quad$ sich in Schweigen hüllen - ćutati

Bd.: Nichts sagen, ruhig sein (gehoben).

\footnotetext{
${ }^{7}$ Im Serbischen wird diese Wortverbindung meistens nicht vollständig, sondern verkürzt bzw. ellipitisch in der Form Schweigen ist Gold verwendet.
} 
W: pokriti/ogrnuti se ćutanjem

Bsp.: Europa darf sich diesbezüglich nicht in Schweigen hüllen.

- dann ist Schweigen im Walde - razgovor se prekida

Bd.: Das Gespräch wurde plötzlich unterbrochen.

W.: tada je tišina u šumi

Bsp.: Wenn das Spiel beginnt, dann ist Schweigen im Walde.

3.3 Die Korpusanalyse resultierte in 18 Beispielen einer partiellen bzw. Teiläquivalenz, von denen elf Phraseologismen morphosyntaktische und lexikalische Unterschiede aufwiesen, während es sich in sieben Fällen ausschließlich um Unterschiede lexikalischer Natur handelte. Die Analyse ergab kein einziges Beispiel einer rein morphosyntaktischen Abweichung. Die folgenden Beispiele zeigen Phraseologismen mit lexikalischen Unterschieden ${ }^{8}$ :

- schweigen wie ein Grab/ein Stein/eine Maus - ćutati kao zaliven

Bd.: Nichts sagen, unter keinen Umständen sprechen, ein Geheimnis bewahren.

Der deutsche Phraseologismus hat im Gegensatz zum Serbischen mehrere Varianten, und zwar mit den Nomen „Grab“, „Stein“ und oder „Maus“. Der lexikalische Unterschied liegt darin, dass der Vergleich im Deutschen mithilfe eines Substantivs angestellt wird (Grab/Stein/Maus), während im Serbischen am Ende steht ein Adjektiv -,,zaliven“ (dt. begossen).

W.: ćutati kao grob/kamen/miš

Bsp.: Ich werde dein Geheimnis nicht verraten. Ich werde schweigen wie ein Grab.

- $\quad$ sich auf die Zunge ${ }^{9}$ beißen - ugristi se (ujesti se) za usnu

Bd.: Etwas absichtlich nicht sagen, was man eigentlich hätte sagen wollen.

Bsp.: Beiß dir lieber auf die Zunge, bevor du deinem Chef deine Meinung sagst.

- ein loses Maul (Mundwerk) haben - biti pogana jezika/imati pogan jezik

8 Im Gegensatz zu den Beispielen, bei derer kontrastiven Analyse Voll- und Nulläquivalenz festgestellt werden konnten, ist das Verhältnis der semantischen Felder „reden“" und „schweigen“" 4:3 zugunsten des zweiten ausgerichtet.

${ }^{9}$ Obwohl der Phraseilogismus ujesti se za jezik im Serbischen gebraucht wird, konnte in den verwendeten Wörterbüchern kein Beispiel von Volläquivalenz gefunden werden. 
Bd.: Frech sein, unhöflich sein. Seine Meinung auch dann äußern, wenn es nicht angebracht ist.

Der Unterschied zwischen Ausgangs- und Zielsprache lässt sich anhand der unterschiedlichen Adjektive und Nomina feststellen, die den Phraseologismus bilden. Während es im Deutschen ein „loses Maul (Mundwerk)“ ist, ist es im Serbischen ,pogan jezik“ (dt. eine schmutzige Zunge).

W.: imati labava usta

Bsp.: Sie hat ein ziemlich loses Mundwerk, das ist oft unangebracht.

- den Mund/das Maul halten - zavezati/začepiti gubicu

Bd.: Ruhig sein, nicht sprechen. Der Unterschied dieser Phraseologismen zeigt sich nur in den unterschiedlichen Verben „halten“ und „zavezati/začepiti“ (dt. zuschnüren/zustöpseln).

W.: držati usta

Bsp.: Du redest nur Blödsinn, halt lieber deinen Mund.

3.4 Die elf Phraseologismen, die morphosyntaktische und lexikalische Unterschiede aufwiesen, sind dem semantischen Feld „reden“ zuzuordnen.

- $\quad$ ein Plappermaul ${ }^{10}$ haben - biti duga jezika

Bd.: Ein geschwätziger Mensch, bzw. jemand, der viel tratscht.

Während im deutschen Phraseologismus ein Akkusativobjekt vorhanden ist, kommt im serbischen der Genitiv vor. Des Weiteren ist auch ein lexikalischer Unterschied festzustellen. Die Zusammensetzung „Plappermaul“ wird im Serbischen durch das Adjektiv „dug“ (serb. lang) und das Nomen ,jezik“ (dt. Zunge) ausgedrückt.

W.: imati laprdaška usta

Bsp.: Warum hast du alles erzählt? Du bist ein echtes Plappermaul.

- über Gott und die Welt reden - govoriti o svemu i svačemu

Bd.: Über alles Mögliche sprechen.

Im deutschen Phraseologismus kommt die Verbindung der Präposition „über“ mit dem Akkusativ vor, während im serbischen der Lokativ ${ }^{11}$ steht.

\footnotetext{
${ }^{10}$ Jemand, der viel plappert, viel redet (ugs. abwertend) (Duden, 2003: 1215).

${ }^{11}$ Der Lokativ beantwortet Fragen wie ,wen?“ und ,worüber?“ und kann einen Ort (an der Uni), eine Zeit (während des Sommers), eine Art und Weise (es fließt wie aus Kannen) oder abstrakte Sachverhalte (sie sprechen über den Sinn des Lebens) bezeichnen. Die
} 
Nebenbei lässt sich auch ein lexikalischer Unterschied feststellen, zumal die im deutschen Phraseologismus vorkommenden Nomina „Gott und die Welt" im serbischen Beispiel mit den Indefinitpronomen „sve i svašta“ ausgedrückt werden.

W.: govoriti o bogu i svetu

Bsp.: Die Professorin redet über Gott und die Welt.

- zum Fenster hinaus reden - govoriti u vetar/uprazno

Bd.: Jemandes Aussage kommt aus irgendeinem Grund nicht beim Empfänger an.

Während im deutschen Phraseologismus der Dativ vorhanden ist, kommt im Serbischen der Akkusativ vor. Lexikalisch betrachtet ist der Unterschied in den folgenden Komponenten zu finden: Das im Deutschen auftretende „Fenster“ kommt im Serbischen als „vetar/uprazno“ (dt. der Wind/ins Leere) vor.

W.: govoriti kroz prozor

Bsp.: Mach dir keine Mühe, du würdest nur zum Fenster hinaus reden.

- um den heißen Brei herumreden - obilaziti kao kiša oko Kragujevca

Bd.: Nicht zum Punkt kommen. Über andere Sachen sprechen, bevor man sich auf das Wesentliche konzentriert.

Auch hier ist ein Unterschied bezüglich des Kasus zu sehen. Im deutschen Phraseologismus kommt der Akkusativ, und im serbischen der Genitiv vor. Im Serbischen ist der Phraseologismus auch durch einen Vergleich geprägt - „kao kiša" (dt. wie der Regen), was im Deutschen nicht der Fall ist. Die Funktion des Syntagma „heißer Brei“ im deutschen Phraseologismus übernimmt im serbischen „Kragujevac“ (eine Stadt in Serbien). Das Verb „herumreden“ kommt im serbischen Phraseologismus nicht vor. Stattdesen wird „obilaziti“ (dt. umgehen) gebraucht.

W.: govoriti oko vruće kaše

Bsp.: Red'nicht um den heißen Brei herum, komm endlich zum Punkt.

Dass sich der morphosyntaktische Unterschied nicht unbedingt in der Kasusverwendung widerspiegelt, zeigen folgende Beispiele:

- wie wenn man Tauben (tauben Ohren) predigt - kao gluvom dobro jutro

deutsche Entsprechung für den serbischen Lokativ wird mit Akkusativ oder Dativ ausgedrückt, wie z. B. über jemanden sprechen und von jemandem sprechen (Đukanović et al., 2006: 609). 
Bd: Jemandem etwas umsonst erzählen, sagen. Das Gespräch geht nur in eine Richtung.

In beiden Phraseologismen ist ein Vergleich vorhanden. Der erste Unterschied lässt sich in der Subjunktion ,wenn“ erkennen, die im serbischen Beispiel nicht vorkommt. In beiden Fällen stehen substantivierte Adjektive „Tauben“ bzw. „gluvom“ im Dativ die, im deutschen Phraseologismus jedoch im Plural und im serbischen im Singular. Das Verb „predigen“ (serb. propovedati) findet im Serbischen eine Entsprechung in der Konstruktion „dobro jutro“ (dt. guten Morgen). Diese fungiert als Subjekt, während ,predigen“ in der Funktion des Prädikats vorkommt.

W: kao kad gluvom propovedaš

Bsp.: Dein Gerede nützt bei ihm nichts. Das ist, wie wenn man Tauben predigt.

- sich mit seiner eigenen Rede schlagen - skočiti sebi u usta

Bd.: Argumente gegen sich selbst äußern.

Das Verb im deutschen Phraseologismus wird reflexiv gebraucht, was auf den serbischen nicht zutrifft. In beiden Phraseologismen kommen Objekte vor, die sowohl lexikalische als auch morphosyntaktische Unterschiede aufweisen. Im deutschen Phraseologismus fungiert das „mit seiner eigenen Rede“ als ein Präpositionalobjekt, während im serbischen Phraseologismus das Pronomen „sebi“ als Dativobjekt vorkommt. Im Serbischen ist auch eine Lokalbestimmung vorhanden, und zwar ,u usta“ (in den Mund).

W: pobediti se sopstvenim govorom

Bsp.: Der Gegner musste gar nichts sagen, er hat sich mit seiner eigenen Rede geschlagen.

- jemandem Rede und Antwort stehen - polagati nekome račune

Bd.: sich bei jemandem rechtfertigen müssen

Im deutschen „Rede und Antwort“ stehen zwei Nomina im Singular, während im Serbischen ein Nomen im Plural vorkommt - „računi“ (dt. Rechnungen). Die morphosyntaktische Entsprechung liegt bei den Pronomina „jemandem“ und „nekome“, die im Dativ stehen. Lexikalisch betrachtet liegt der Unterschied zwischen „Rede“ und „računi“, aber auch im Verb „stehen“, an wessen Stelle im serbischen Phraseologismus das Verb „polagati“ (dt. ablegen) steht.

W: nekome stajati govor i odgovor

Bsp.: Ich hab einen Fehler gemacht, also muss ich Rede und Antwort stehen. 


\section{SCHLUSSFOLGERUNG}

In diesem Beitrag wurden serbische und deutsche Phraseologismen im semantischen Feld „reden“ und „schweigen“ analysiert. Dabei wurde das Deutsche als Ausgangs- und das Serbische als Zielsprache betrachtet. Anhand der Methode der interlingualen kontrastiven Analyse wurden insgesamt 49 Phraseologismen beschrieben, die verschiedenen einsprachigen und zweisprachigen phraseologischen Wörterbüchern beider Sprachen entnommen wurden, wobei es sich um folgende handelt: Wer hat den Teufel an die Wand gemalt? vom Dudenverlag, Deutsch-serbokroatisches phraseologisches Wörterbuch von Pavica Mrazović und Ružica Primorac, Hrvatsko-njemački frazeološki rječnik und Frazeološki rječnik hrvatskoga ili srpskog jezika von Josip Matešić, Srpskonemački prevodni frazeološki rečnik von Božinka Petronijević, Lexikon der sprichwörtlichen Redensarten von Lutz Röhrich und Frazeološki rečnik srpskog jezika von Đorđe Otašević.

Von den insgesamt 49 Phraseologismen sind 36 dem semantischen Feld „reden“ und zwölf dem semantischen Feld „schweigen“ zuzuschreiben. Ein Beispiel erwies sich als gemeinsamer Auftritt, und zwar: Reden ist Silber, Schweigen ist Gold. Geleitet von der Klassifizierung von Pavica Mrazović kam man zum folgenden Ergebnis:

Von den 49 Phraseologismen stellten sich 15 als volläquivalent heraus, während 16 Nulläquivalenz aufwiesen. Die restlichen 18 haben im Serbischen nur Teiläquivalente, wobei bei sieben nur lexikalische und bei elf lexikalische und morphosyntaktische Unterschiede ermittelt werden konnten. Die Analyse ergab keine Beispiele für „falsche Freunde“ oder partielle Äquivalenz mit ausschließlich morphosyntaktischen Unterschieden. In den sieben Phraseologismen, in denen nur lexikalische Unterschiede vorhanden waren, wurde ein Wechselspiel der Lexeme Mund/Maul, Zunge und Lippe bemerkt, dabei tritt am häufigsten die Situation auf, in der die deutsche Variante mit Mund/Maul im Serbischen Zunge (serb. jezik) oder Lippe (serb. usna) lautet. Das Lexem Zunge tritt auch im Deutschen auf, Lippe kommt jedoch nur im Serbischen vor. Die Beispiele mit morphosyntaktischen und lexikalischen Unterschieden wiesen darauf hin, dass in den deutschen Phraseologismen meistens der Dativ vorkam, während im Serbischen keine Dominanz eines bestimmten Kasus festgestellt werden konnte. Es wurde ebenfalls festgestellt, dass unter den deutschen Phraseologismen auch mehrere Varianten desselben Phraseologismus vorkommen können (,eine 
böse/spitze/scharfe/giftige Zunge haben“), was im Serbischen nicht der Fall ist (,imati oštar jezik“).

\section{LITERATURVERZEICHNIS}

Burger, Harald et al. 1982. Handbuch der Phraseologie. Berlin/New York: Walter de Gruyter.

Burger, Harald. 2003. Phraseologie: Eine Einführung am Beispiel des Deutschen. 2. überarbeitete Auflage. Berlin: Erich Schmidt Verlag.

Duden. 2003. Deutsches Universalwörterbuch. 5., überarbeitete Aufl. Mannheim: Dudenverlag.

Duden. 2014. Wer hat den Teufel an die Wand gemalt?. Berlin: Dudenverlag.

Fleischer, Wolfgang. 1997. Phraseologie der deutschen Gegenwartssprache. 2. durchgesehene und ergänzte Auflage. Tübingen: Max Niemeyer Verlag.

Korhonen, Jarmo. 2007. „Phraseme im Sprachgebrauch und in der Übersetzung“. In: Phraseologie - Ein internationales Handbuch der zeitgenössischen Forschung. Berlin/New York: Walter de Gruyter: 574-589.

Matešić, Josip. 1982. Frazeološki rječnik hrvatskoga ili srpskog jezika. Zagreb: Školska knjiga.

Matešić, Josip. 1988. Hrvatsko-njemački frazeološki rječnik. Zagreb: Nakladni zavod Matice hrvatske.

Mrazović, Pavica; Primorac, Ružica. 1964-1965. „Pokušaj klasifikacije frazeoloških izraza nemačkog jezika, njihovi izrazi i stilske funkcije“. In: Godišnjak Filozofskog fakulteta. Heft 8, Novi Sad: Filozofski fakultet.

Mrazović, Pavica; Primorac, Ružica. 1981. Nemačko-srpskohrvatski frazeološki rečnik. Beograd: Narodna knjiga.

Otašević, Đorđe. 2012. Frazeološki rečnik srpskog jezika. Novi Sad: Prometej.

Palm, Christine. 1997. Phraseologie. Eine Einführung. Tübingen: Gunter Narr Verlag.

Petronijević, Božinka. 2007. Srpsko-nemački prevodni frazeološki rečnik. Beograd: Jasen.

Röhrich, Lutz. 2004. Lexikon der sprichwörtlichen Redensarten. Bd. 3, Salamander-Zylinder. Freiburg: Herd.

Ђукановић и др. 2006. Основни речник немачко-српски српско-немачки: са немачком и српском граматиком. Београд: Завод за уџбенике. 
Aleksandar Nikolić

CONTRASTIVE ANALYSIS OF GERMAN PHRASEOLOGISMS IN THE SEMANTIC FIELD „TALKING“ AND „BEEING SILENT“ AND THEIR EQUIVALENTS IN SERBIAN

\section{Summary}

The following paper deals with German idioms from the semantic field "talking" and "being silent" and their corresponding equivalents in Serbian. Not only examples with the components "talking" and "being silent" come into question, but also all idioms from whose meaning "talking" and "being silent" can be inferred. The corpus consists of a total of 49 examples from various monolingual and bilingual phraseological dictionaries in both languages. The aim of this paper is to use the method of interlingual contrastive analysis to examine all idioms for morphological, morphosyntactic and lexical similarities and differences in order to assign them accordingly to the equivalence types of Pavica Mrazović. The analysis of the 49 idioms has shown 15 idioms with full equivalence, 7 with lexical differences, 11 with morphosyntactic and lexical differences and 16 examples in which there is no equivalent in Serbian.

Keywords: talking, being silent, idiom, German, Serbian 